\title{
Mobility edge and locality of the overlap-Dirac operator with and without dynamical overlap fermions
}

\author{
JLQCD Collaboration: N. Yamada ${ }^{* a, b \dagger}$, S. Aoki ${ }^{c, d}$, H. Fukaya ${ }^{e}$, S. Hashimoto ${ }^{a, b}$, \\ K-I. Ishikawa ${ }^{f}$, K. Kanaya ${ }^{c}$, T. Kaneko ${ }^{a, b}$, H. Matsufuru ${ }^{a}$, M. Okamoto ${ }^{a}$, T. Onogi ${ }^{g}$ \\ ${ }^{a}$ High Energy Accelerator Research Organization (KEK), Tsukuba 305-0801,Japan \\ ${ }^{b}$ School of High Energy Accelerator Science, The Graduate University for Advanced Studies \\ (Sokendai), Tsukuba 305-0801, Japan \\ ${ }^{c}$ Graduate School of Pure and Applied Sciences, University of Tsukuba, Tsukuba 305-8571, \\ Japan \\ ${ }^{d}$ Riken BNL Research Center, Brookhaven National Laboratory, Upton, New York 11973, USA \\ ${ }^{e}$ Theoretical Physics Laboratory, RIKEN, Wako 351-0198, Japan \\ ${ }^{f}$ Department of Physics, Hiroshima University, Higashi-Hiroshima 739-8526, Japan \\ ${ }^{g}$ Yukawa Institute for Theoretical Physics, Kyoto University, Kyoto 606-8502, Japan
}

\begin{abstract}
We perform a systematic study of low-lying eigenmodes of $H_{w}$ with various gauge actions to find the optimal choice for dynamical overlap fermion simulations, with which one may achieve lower numerical cost for HMC and better locality property of the overlap kernel. For this purpose, our study is made with emphasis on the distribution of low-lying eigenvalues and the mobility edge with and without dynamical overlap fermions.
\end{abstract}

XXIV International Symposium on Lattice Field Theory

July 23-28 2006

Tucson Arizona, US

\footnotetext{
*Speaker.

${ }^{\dagger}$ E-mail: norikazu.yamada@kek.jp
} 


\section{Introduction}

The JLQCD collaboration has started a large scale simulation of dynamical overlap fermions [1], aiming at studying the QCD dynamics in the presence of very light quarks. For the overlap fermion formulation, large computational costs and non-locality of the overlap-Dirac operator have been practical and theoretical concerns. The computational cost is closely related to the near-zero mode density of the Hermitian Wilson-Dirac operator,

$$
H_{\mathrm{w}}\left(-m_{0}\right)=\gamma_{5} D_{\mathrm{w}}\left(-m_{0}\right)=\gamma_{5}\left(-m_{0}+D_{\mathrm{w}}(0)\right) \quad\left(m_{0}=1+s\right),
$$

where $D_{\mathrm{w}}(0)$ is the standard Wilson-Dirac operator for the massless Wilson fermion. According to Ref. [2], locality of the overlap-Dirac operator can be studied by examining the locality of eigenvectors of $H_{\mathrm{w}}$, defined by $H_{\mathrm{w}} \phi_{i}(x)=\lambda_{\mathrm{w}, i} \phi_{i}(x)$. Since both the near-zero mode density and the corresponding eigenvectors are known to depend on the gauge action and $\beta$, in this work we explore these two properties for several gauge actions.

We consider three gauge actions, the standard plaquette (Plq), Iwasaki (RG), and a modified gauge action motivated by the admissibility condition (Adm). The Adm action [3, 4, 5] is given by

$$
S_{\mathrm{Adm}}=\left\{\begin{array}{cl}
\beta \sum_{x, \mu, v} \frac{1-\operatorname{Re} \operatorname{Tr} P_{\mu, v}(x) / 3}{1-\left(1-\operatorname{Re} \operatorname{Tr} P_{\mu, v} / 3\right) / \varepsilon}, & \text { when } 1-\operatorname{Re} \operatorname{Tr} P_{\mu, v} / 3<\varepsilon \\
\infty & \text { otherwise }
\end{array},\right.
$$

where $\varepsilon$ is a parameter to control the possible maximum value of $\operatorname{Re} \operatorname{Tr} P_{\mu, v}(x) / 3$. With $\varepsilon<$ $1 /(6(2+\sqrt{2}))$, the locality of the overlap-Dirac operator is guaranteed [6]. This action reduces to the standard plaquette action when $1 / \varepsilon \rightarrow 0$. In addition, for each of these gauge actions we introduce two-flavors of extra-Wilson fermions $\psi$ and ghosts $\chi[7]$ as

$$
S_{\mathrm{ext}}=\sum_{x} \bar{\psi}(x) D_{\mathrm{w}}\left(-m_{0}\right) \psi(x)+\sum_{x} \chi^{\dagger}(x)\left(D_{\mathrm{w}}\left(-m_{0}\right)+i \mu \gamma_{5} \tau^{3}\right) \chi(x),
$$

where $\tau^{3}$ acts on the flavor index. Since integrating out these extra fields results in $\operatorname{det} \mid H_{\mathrm{w}}^{2}\left(-m_{0}\right)$ $/\left(H_{\mathrm{w}}^{2}\left(-m_{0}\right)+\mu^{2}\right) \mid$, the appearance of the modes with $\left|\lambda_{\mathrm{w}, i}\right|<\mu$ are suppressed with this weight. $\mu=0$ corresponds to the standard quenched approximation. The same value of $m_{0}$ is taken for both the overlap kernel and extra fields.

The lattice size is fixed to $16^{3} \times 32$. For the Adm action, $1 / \varepsilon=2 / 3$ is used throughout this study. Three values of $\mu(0.0,0.2$, and 0.4$)$ are examined, and more than 10,000 trajectories are accumulated for each gauge action as shown in Tab. 1. The lattice spacing is set by $r_{0}=0.49 \mathrm{fm}$, and is about $0.125 \mathrm{fm}$ for all lattices, unless otherwise stated.

\section{Spectral density}

Three panels in Fig. 1 show the distributions of the near-zero modes for three gauge actions, where all eigenvalues are plotted in the lattice unit. Different symbols represent different values of $\mu$. We find that the extra fermions and ghosts suppress the low-lying modes as expected. The suppression is most effective with $\mu=0.2$. Figure 2 shows a comparison of the spectral density $\rho\left(\lambda_{\mathrm{w}}\right)$ for the three gauge action with $\mu=0.2$. We observe that the $\mathrm{RG}$ action yields the smallest near-zero mode density. From these observations made in the quenched approximation, we decide to employ the Iwasaki RG gauge action with $\mu=0.2$ in the dynamical overlap simulations. 


\begin{tabular}{c|cccc} 
action & $\beta$ & $\mu$ & $1 / \varepsilon$ & \# of trj. \\
\hline Plq & 5.83 & 0.0 & & 20,000 \\
& 5.70 & 0.2 & & 11,600 \\
& 5.45 & 0.4 & & 11,600 \\
\hline RG & 2.43 & 0.0 & & 20,000 \\
& 2.37 & 0.2 & & 21,600 \\
& 2.27 & 0.4 & & 20,000 \\
\hline Adm & 2.33 & 0.0 & $2 / 3$ & 20,000 \\
& 2.23 & 0.2 & $2 / 3$ & 20,000 \\
& 2.06 & 0.4 & $2 / 3$ & 14,800
\end{tabular}

Table 1: Simulation parameters.
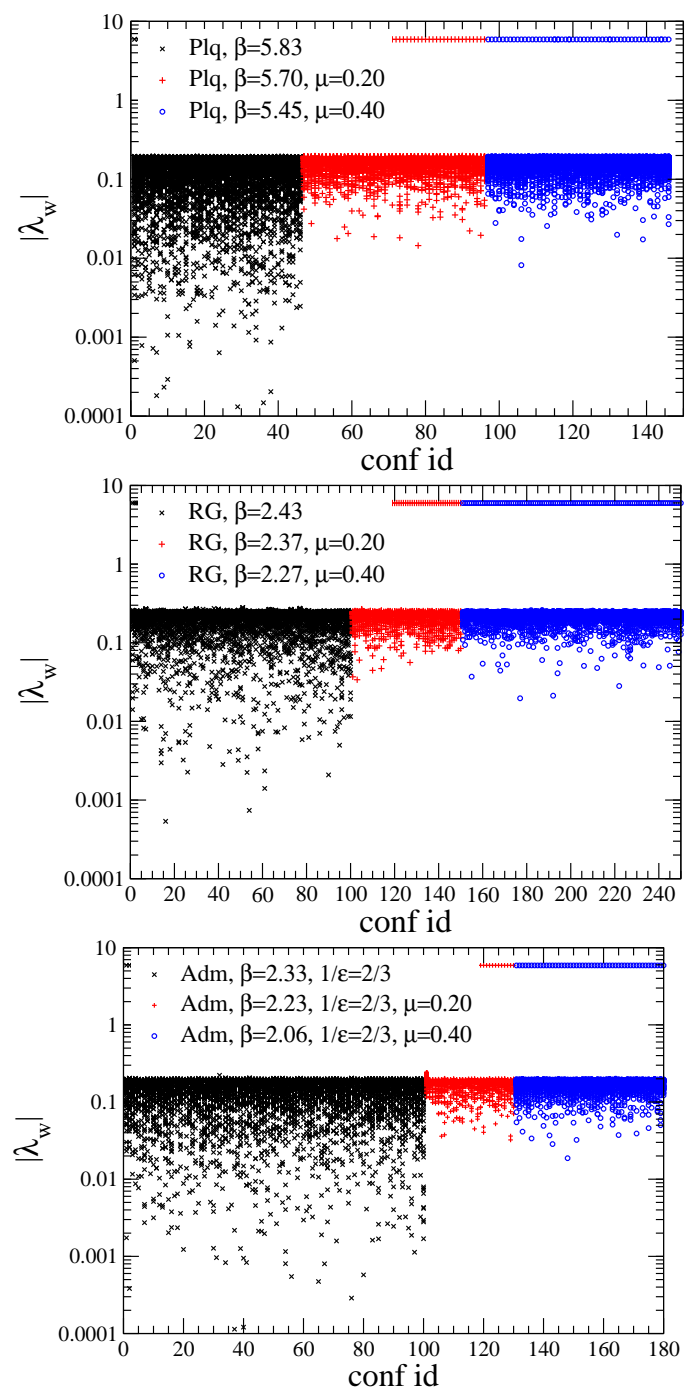

Figure 1: Distribution of near-zero modes for three different gauge actions, Plq, RG and Adm from top to bottom, and with three values of $\mu=0.0,0.2,0.4$ from left to right.

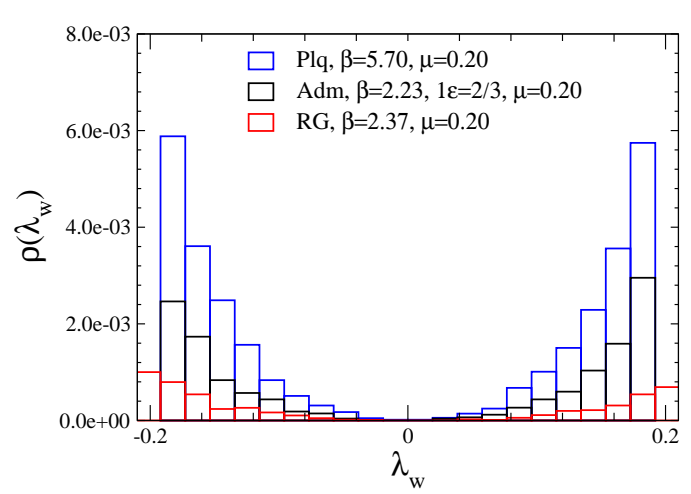

Figure 2: Spectral densities for three quenched gauge actions, Plq (blue), Adm (black) and RG (red) with $\mu=0.2$.

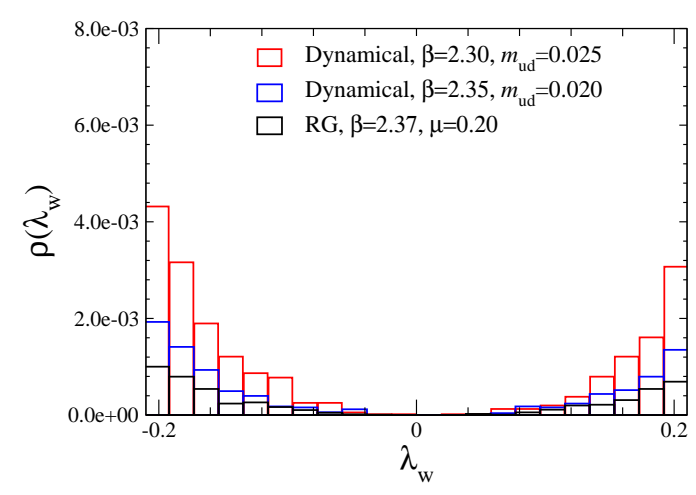

Figure 3: Comparison of the near-zero mode densities on the quenched (black), slightly fine (blue) and coarse (red) dynamical configurations.

We made the similar study on dynamical configurations, and compare the results with that on the quenched results with $\mu=0.2$ in Fig. B. The results from two dynamical ensembles with $\beta=2.30$ and 2.35 are shown, their lattice spacings are $a=0.12 \mathrm{fm}$ and $0.11 \mathrm{fm}$, respectively, and the sea quark mass is about $m_{s} / 4$ in both cases. While the density increases for the dynamical configurations, it is clear that the nearzero mode suppression works well even after incorporating the overlap sea quarks. More details on our dynamical configurations are found in Ref. [1].

\section{Mobility edge}

The overlap-Dirac operator is proved to be exponentially local, if there is no low-lying mode 
below some threshold [8]. In practice, the density of the near-zero mode is non-zero, unless we introduce the determinant factor such as $\operatorname{det} H_{\mathrm{w}}^{2}$. Even in this case, the overlap-Dirac operator is exponentially local if the near-zero modes themselves are exponentially localized [2]. Golterman, Shamir, and Svetitsky argued that the magnitude of the overlap operator behaves as

$$
\left|D_{\text {ov }}(x, y)\right| \sim \bar{\lambda} \rho(\bar{\lambda}) \exp \left(-\frac{|x-y|}{2 l_{l}(\bar{\lambda})}\right)+O(1) \cdot \exp \left(-\lambda_{c}|x-y|\right),
$$

where $\bar{\lambda}$ denotes a near-maximum eigenvalue of low-lying localized modes (somewhat ambiguous), $l_{l}(\bar{\lambda})$ a localization length at $\bar{\lambda}$. The parameter $\lambda_{c}$ stands for the mobility edge, which separates localized and extended modes. The first term is derived from the dependence of $\rho(\lambda)$ and $l_{l}(\lambda)$ on $\lambda$, while the second term is a conjecture motivated by numerical experiences [2]. In most cases, it is known that the second term dominates the first.

Mobility edge is determined from the eigenvectors as follows. We first define $\rho_{i}(x)$ and $f_{i}(r)$ as

$$
\begin{aligned}
\rho_{i}(x) & =\phi_{i}^{\dagger}(x) \phi_{i}(x), \quad \rho_{i}\left(x_{0}\right)=\max _{x}\left\{\rho_{i}(x)\right\} \\
f_{i}(r) & =\left\{\bar{\rho}_{i}(x)|r=| x-x_{0} \mid\right\}
\end{aligned}
$$

where $\bar{\rho}_{i}(x)$ is an average of $\rho_{i}(x)$ over the lattice points which have the same distance $r$ from $x_{0}$. The eigenvalues $\left|\lambda_{\mathrm{w}, \mathrm{i}}\right|$ are binned with a certain bin size, and $f_{i}(r)$ is averaged over the modes within a bin. The localization length at each bin, $l_{l}\left(\lambda_{\mathrm{w}, \mathrm{i}}\right)$, is then obtained at large $r$ by fitting to

$$
f_{i}(r)=\exp \left(-\frac{r}{l_{l}\left(\lambda_{\mathrm{w}, \mathrm{i}}\right)}\right) \text {. }
$$

Mobility edge, $\lambda_{c}$, is defined by $\lambda_{\mathrm{w}, \mathrm{i}}$ at which $l_{l}\left(\lambda_{\mathrm{w}, \mathrm{i}}\right)$ diverges. Figure $\bigoplus$ shows an example of $f_{i}(r)$ obtained on a single configuration. We can clearly see that the decay rate becomes smaller for larger eigenvalues.

The determination of $\lambda_{c}$ is performed with a fixed value of $m_{0}=1.6$. In addition to the ensembles used in the study of spectral density, the determination is performed on three coarse lattices, which are generated with the Plq action with $\beta=5.70$ and 5.40 and the $\mathrm{RG}$ action with $\beta=2.43$, to see the dependence on lattice spacing. $l_{l}\left(\lambda_{\mathrm{w}, i}\right)$ extracted from the fit using eq. (3.4) is plotted as a

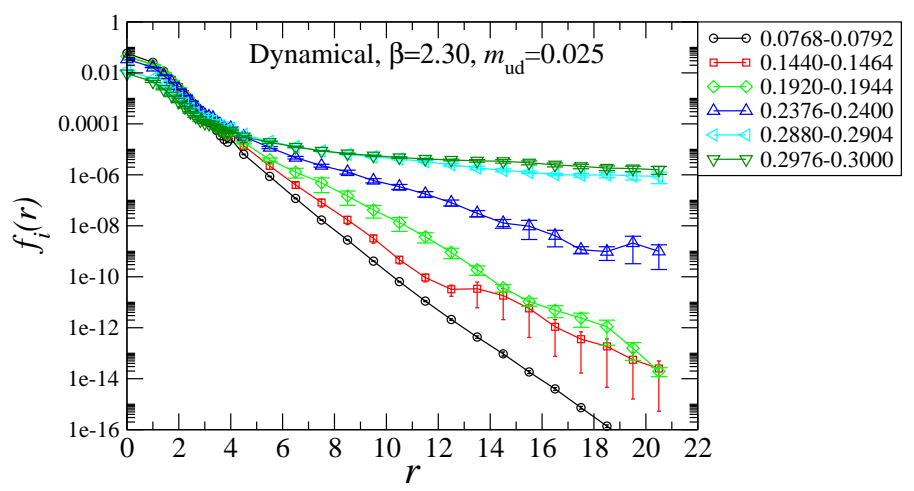

Figure 4: Example of $f_{i}(r)$. Different symbols represent different bin in $\lambda_{\mathrm{w}}$, as denoted in the plots. Data for the dynamical configuration at $\beta=2.30, m_{\text {sea }}=0.025$. 

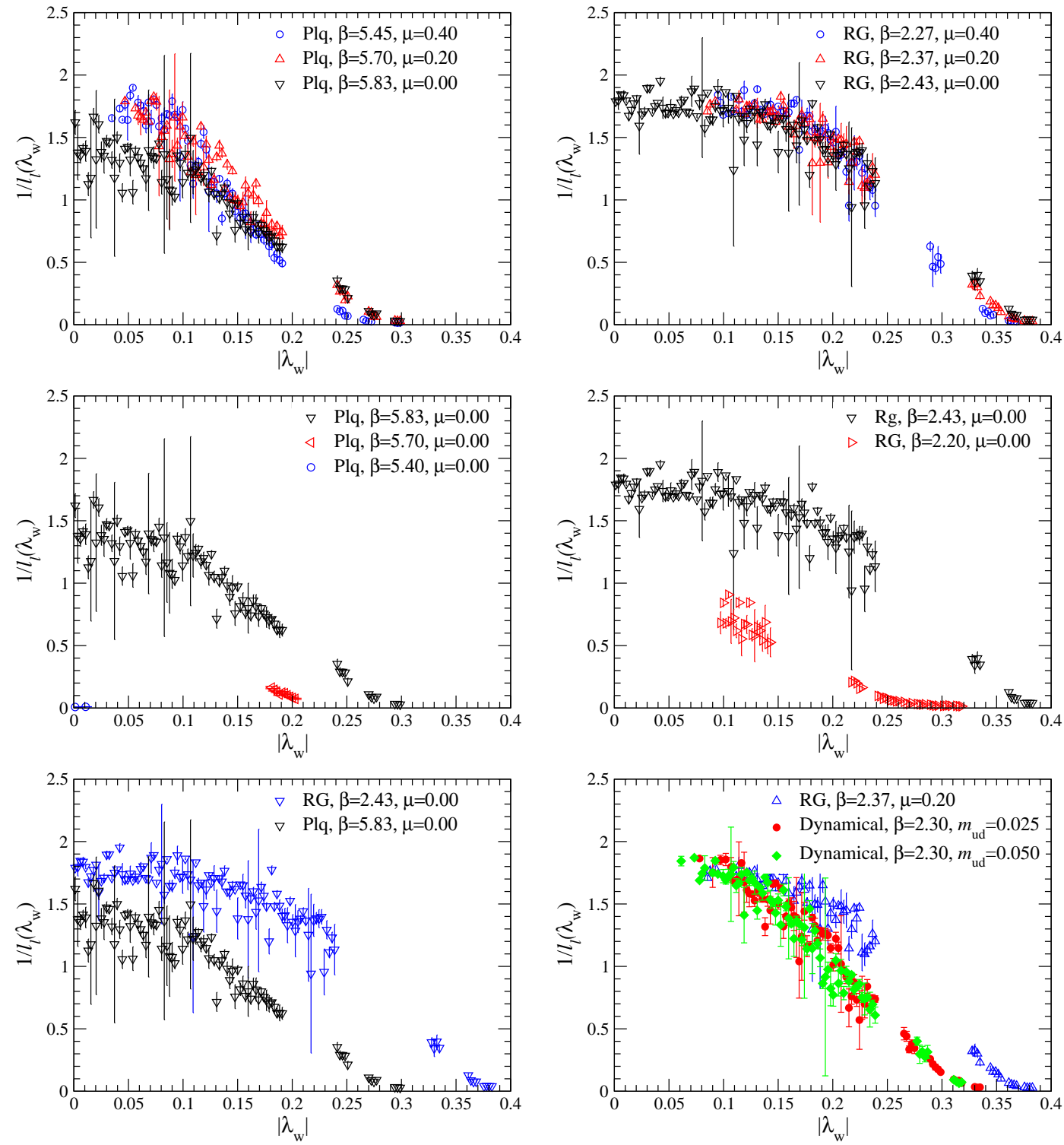

Figure 5: Inverse localization length $1 / l_{l}\left(\lambda_{\mathrm{w}}\right)$ as a function of $\left|\lambda_{\mathrm{w}}\right|$.

function of $\lambda_{\mathrm{w}, i}$ in Fig. 5 for various ensembles. In the two plots at the top of Fig. 5 the results at the same lattice spacing, $a=0.125 \mathrm{fm}$, but with different $\mu$ are plotted for the Plq (left) and the RG (right). $\lambda_{c}$ is found to be $0.24-0.27$ for the plaquette action and $0.34-0.36$ for the RG action in the lattice unit. Dependence of $\lambda_{c}$ on $\mu$ turns out to be weak in the range of $0.0<\mu<0.4$.

From the two plots in the middle showing the results with $\mu=0$ for two or three different $\beta$, we find that $\lambda_{c}$ in the lattice unit decreases as $\beta$ decreases. The data at $\beta=5.40$ for the plaquette action (circle in the left-middle panel) shows $1 / l_{l}(0) \sim 0$. Ref. [2] suggests to use " $1 / l_{l}(0)=0$ " as the definition of the Aoki phase.

In the left-bottom, the results with the plaquette and RG actions are compared at the same lattice spacing. Clearly the RG action gives lager $\lambda_{c}$ than that of the Plq action. Finally, the right-bottom figure shows a comparison of the results from quenched and dynamical runs with two different sea quark masses. Here we do not see any significant difference between the quenched 


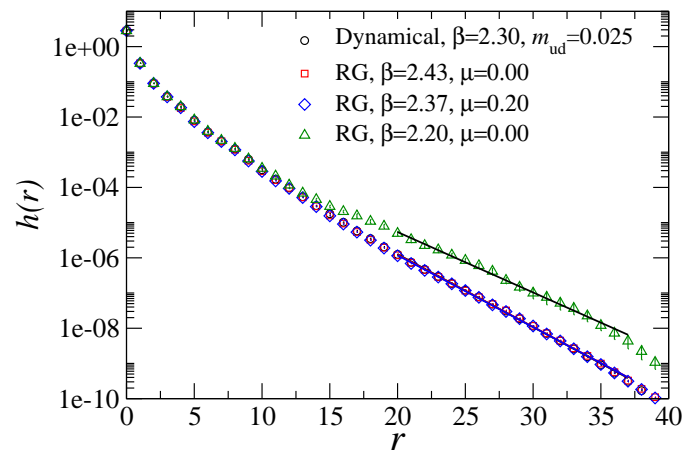

Figure 6: $r$ dependence of $h(r)$ for four different ensembles.

and dynamical runs nor clear dependence on sea quark mass within dynamical runs. $\lambda_{c}$ turns out to be $500-600 \mathrm{MeV}$ in our dynamical lattices.

We also measure $1 / l_{\text {ov }}$ from the $r$ dependence of $h(r)$ defined by

$$
h(r)=\max _{x}\left\{\left\|D_{\mathrm{ov}}(x, y) \delta\left(y-x_{0}\right)\right\|\left|r=\sum_{\mu}\right| x_{\mu}-x_{0 \mu} \mid\right\} .
$$

$h(r)$ is measured on the same ensembles, and some of the results obtained with the RG action are plotted in Fig. 6, where numerical data are in the lattice unit. We see that all results except for the one with $\beta=2.20$ coincide with each other. $\beta=2.20$ corresponds to $a \approx 0.2 \mathrm{fm}$, while others are $a=0.120-0.125 \mathrm{fm}$. By fitting this to the same form as eq. (3.4), we obtain $1 / l_{\mathrm{ov}}$. For our dynamical lattice at $\beta=2.30,1 / l_{\mathrm{ov}}$ is estimated to be about $800 \mathrm{MeV}$.

In Fig. 7, $\lambda_{c}$ and $1 / l_{\mathrm{ov}}$ obtained with the RG action are compared. While both $1 / l_{\mathrm{ov}}$ and $\lambda_{c}$ show the similar dependence on lattice spacing, the difference is sizable. Within the range of the lattice spacing we have studied it turns out that $1 / l_{\mathrm{ov}}>\lambda_{c}$. Although $\lambda_{c} \sim 500-600 \mathrm{MeV}$ in our dynamical lattice is not much larger than $\Lambda_{\mathrm{QCD}}, 1 / l_{\mathrm{ov}} \sim 800 \mathrm{MeV}$ is probably acceptable for simulations of QCD. In Ref. [9], $1 / l_{\mathrm{ov}}$ has been studied at several different lattice spacings in the range of $a \sim 0.13-0.20 \mathrm{fm}$ within the quenched approximation. They reported acceptably large values for $1 / l_{\mathrm{ov}}$ at all lattices, which appears to be consistent with our observation.

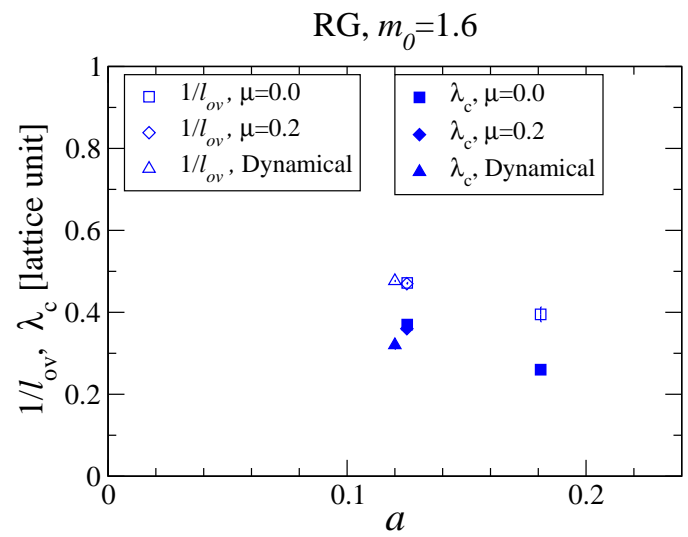

Figure 7: $a$ dependence of $1 / l_{\mathrm{ov}}$ and $\lambda_{c}$. 


\section{Summary}

In this work, near-zero mode density for various gauge actions are examined from the viewpoint of cost reduction in dynamical overlap simulation, and the RG Iwasaki action with extra fermions and ghosts exhibits smallest near-zero mode density among the actions studied. We also determine the mobility edge and the localization range of the overlap-Dirac operator. While $\lambda_{c}$ and $1 / l_{\mathrm{ov}}$ seem to agree with each other qualitatively, the difference is sizable. $1 / l_{\mathrm{ov}}$ tells us the locality of an given overlap-Dirac operator and is presumably all we need to know from the practical point of view, but the precise relationship between $1 / l_{\mathrm{ov}}$ and $\lambda_{c}$ should be understood.

\section{Acknowledgment}

Numerical simulations are performed on Hitachi SR11000 and IBM System Blue Gene Solution at High Energy Accelerator Research Organization (KEK) under a support of its Large Scale Simulation Program (No. 06-13). This work is supported in part by the Grant-in-Aid of the Ministry of Education (Nos. 13135204, 13135213, 15540251, 16740147, 16740156, 17340066, 17740171, 18034011, 18340075, 18740167.)

\section{References}

[1] H. Fukaya et al. [JLQCD collaboration], PoS LAT2006, 050 (2006); S. Hashimoto et al. [JLQCD collaboration], PoS LAT2006, 052 (2006); T. Kaneko et al. [JLQCD collaboration], PoS LAT2006, 054 (2006); H. Matsufuru et. al., [JLQCD collaboration], PoS LAT2006, 031 (2006).

[2] M. Golterman and Y. Shamir, Phys. Rev. D 68, 074501 (2003) [arXiv:hep-lat/0306002]; M. Golterman, Y. Shamir and B. Svetitsky, Phys. Rev. D 71, 071502 (2005) [arXiv:hep-lat/0407021]; Phys. Rev. D 72, 034501 (2005) [arXiv:hep-lat/0503037].

[3] M. Luscher, Nucl. Phys. B 549, 295 (1999) [arXiv:hep-lat/9811032].

[4] H. Fukaya, S. Hashimoto, T. Hirohashi, K. Ogawa and T. Onogi, Phys. Rev. D 73, 014503 (2006) [arXiv:hep-lat/0510116].

[5] W. Bietenholz, K. Jansen, K. I. Nagai, S. Necco, L. Scorzato and S. Shcheredin, JHEP 0603, 017 (2006) [arXiv:hep-lat/0511016].

[6] H. Neuberger, Phys. Rev. D 61, 085015 (2000) [arXiv:hep-lat/9911004].

[7] T. Izubuchi and C. Dawson [RBC Collaboration], Nucl. Phys. Proc. Suppl. 106, 748 (2002); M. Luscher, private communication; H. Fukaya, arXiv:hep-lat/0603008; P. M. Vranas, Phys. Rev. D 74, 034512 (2006) [arXiv:hep-lat/0606014]; in these proceedings; H. Fukaya, S. Hashimoto, K. I. Ishikawa, T. Kaneko, H. Matsufuru, T. Onogi and N. Yamada [JLQCD Collaboration], arXiv:hep-lat/0607020.

[8] P. Hernandez, K. Jansen and M. Lüscher, Nucl. Phys. B 552, 363 (1999) [arXiv:hep-lat/9808010].

[9] T. Draper et al., PoS LAT2005, 120 (2006) [arXiv:hep-lat/0510075]; T. Draper et al., arXiv:hep-lat/0609034. 